\title{
Subacute Thyroiditis during the COVID-19 Pandemic: Searching for a Clinical Association with SARS-CoV-2
}

\author{
Pierpaolo Trimboli $\left(\mathbb{D},{ }^{1,2}\right.$ Chiara Camponovo $\left(\mathbb{D},{ }^{1}\right.$ Sebastiano Franscella $\left(\mathbb{D},{ }^{1}\right.$ \\ Enos Bernasconi $\mathbb{D}^{2,3}$ and Niccolò Buetti ${ }^{40}{ }^{4,5,6}$ \\ ${ }^{1}$ Clinic for Endocrinology and Diabetology, Lugano Regional Hospital, Ente Ospedaliero Cantonale, Lugano, Switzerland \\ ${ }^{2}$ Università della Svizzera Italiana (USI), Lugano, Switzerland \\ ${ }^{3}$ Division of Infectious Diseases, Lugano Regional Hospital, Ente Ospedaliero Cantonale, Lugano, Switzerland \\ ${ }^{4}$ Ente Ospedaliero Cantonale, Locarno Regional Hospital, Locarno, Switzerland \\ ${ }^{5}$ University of Paris, INSERM IAME, U1137, Team DeSCID, Paris, France \\ ${ }^{6}$ Infection Control Program and World Health Organization Collaborating Centre on Patient Safety, \\ University Hospitals and Faculty of Medicine, University of Geneva, Geneva, Switzerland
}

Correspondence should be addressed to Pierpaolo Trimboli; pierpaolo.trimboli@eoc.ch

Received 10 February 2021; Revised 5 March 2021; Accepted 12 March 2021; Published 26 March 2021

Academic Editor: Alberto Ferlin

Copyright (c) 2021 Pierpaolo Trimboli et al. This is an open access article distributed under the Creative Commons Attribution License, which permits unrestricted use, distribution, and reproduction in any medium, provided the original work is properly cited.

\begin{abstract}
Purpose. To search for a clinical potential link between subacute thyroiditis (SAT) and severe acute respiratory syndrome coronavirus 2 (SARS-CoV-2) in a series of patients diagnosed with SAT during the COVID-19 pandemic, by retrospective evaluation of (1) clinical symptoms and (2) contact tracing. Methods. SAT patients diagnosed from March to December 2020 were enrolled. The presence of typical clinical presentation of SARS-CoV-2, diagnostic tests for SARS-CoV-2, and contact with other individuals proven to be positive for SARS-CoV-2 were searched. Results. Ten SAT cases were included. Fever was recorded in four patients. Cough, dyspnea, and headache were rarely reported. No patient had diagnosis of pneumonia. Two patients had moderate to severe fatigue after SAT. One patient experienced loss of smell and taste and had persistent fatigue over the following five months. No patient had positive SARS-CoV-2 diagnostic tests. At contact-tracing evaluation, only one patient had a contact with people who were diagnosed with SARS-CoV-2. Conclusions. Patients diagnosed with SAT during COVID-19 pandemic rarely experienced SARS-CoV-2-related symptomatology. The contact tracing did not show close contact with SARS-CoV-2 individuals in $9 / 10$ cases.
\end{abstract}

\section{Introduction}

In March 2020, the World Health Organization declared the pandemic of novel coronavirus disease 2019 (COVID-19) caused by the severe acute respiratory syndrome coronavirus 2 (SARS-CoV-2) [1]. Since then, more than 70 million cases with more than 1.5 million deaths from SARS-CoV-2 were reported until December 2020 [2]. Coronaviruses are known to have a direct effect on endocrine glands and a direct damage by SARS-CoV to the thyroid follicular and parafollicular cells was demonstrated in vitro in 2007 [3]. During 2020, with the worldwide rapid diffusion of COVID19 , several data on the relationship between SARS-CoV-2 and thyroid disease have been emerging [4]. Some of these studies focused on the subacute thyroiditis (SAT), also known as De Quervain's thyroiditis, a self-limited inflammatory thyroid disease caused by several viruses and usually preceded by upper respiratory tract infections [5]. The clinical presentation of SAT encompasses neck pain, as the hallmark of the clinical syndrome, thyroid enlargement, 
general symptoms (e.g., mild fever and fatigue), and thyroid dysfunction. Classically, the clinical course of SAT includes three consecutive 2-month phases: (1) thyrotoxicosis due to the parenchymal damage; (2) hypothyroidism due to the deficiency of hormones during the initial restoring of parenchyma; (3) euthyroidism with the restitutio ad integrum of parenchyma [6]. Many viruses are known to be associated with the development of SAT, while a direct evidence of the presence of virus in the thyroid tissue is available only for a few cases [5]. In a recent full review of the literature about the impact of COVID-19 on the thyroid gland [4], the published cases of SAT during the COVID-19 pandemic were detailed. Although several clues could lead to a possible relationship between SAT and SARS-CoV-2, and the presence of the receptor for SARS-CoV-2 entry (i.e., angiotensinconverting enzyme 2 (ACE-2)) in the thyroid cells has been demonstrated [7]; a direct clinical association between SAT and SARS-CoV-2 has not been clarified.

The aim of the present study was to investigate the possible clinical relationship between SAT and SARS-CoV-2 in a series of patients diagnosed with SAT during the ongoing pandemic by retrospective (1) evaluation of the presence of typical symptoms of SARS-CoV-2 and (2) reconstruction of the history of contacts with other individuals diagnosed with SARS-CoV-2.

\section{Methods}

The study was conducted at the Clinic for Endocrinology and Diabetology of Lugano Regional Hospital located in the Italian-speaking region of Switzerland (Canton Ticino). In this region, two COVID-19 pandemic waves were observed, the first started in March and the second in October 2020.

All patients diagnosed with SAT during the period from March $1^{\text {st }}$ to December $15^{\text {th }}, 2020$, and recorded at our Clinic were enrolled in the study. The patients' clinical history was systematically searched for the presence of typical symptoms and signs of SARS-CoV-2 (i.e., fever, cough, dyspnea, headache, fatigue, pneumonia, loss of smell, and loss of taste) and they were classified according to onset's time of SAT (i.e., before, concomitant, or after). Specific reverse transcriptase polymerase chain reaction (rtPCR) in nasopharyngeal swabs and serum diagnostic tests for SARS-CoV-2 were recorded, when performed. The reconstruction of contact tracing of the included cases was conducted in December 2020 by a patients' interview to achieve information on their contact with other individuals proven to be positive for SARS-CoV-2 during the period between March to December 2020.

\section{Results}

The series included 10 patients ( 9 females and 1 male, mean age 47 years). The course of SAT was favorable in all cases with recovery. A low-dose (i.e., $20 \mathrm{mg}$ per day) prednisone therapy was administered.

The most common symptoms of SARS-CoV-2 were searched and detailed in Table 1. Fever was recorded in five patients with no high body temperature and was concomitant with SAT. Cough, dyspnea, and headache were rarely experienced. No patient had diagnosis of pneumonia. Two patients had moderate to severe fatigue after SAT. One patient (\#9) experienced loss of smell and taste after SAT and had persistent fatigue over the following five months. Four patients underwent SARS-CoV-2 nasopharyngeal swab while SARS-CoV-2 serum test was performed in two cases; no patient had positive SARS-CoV-2 diagnostic tests.

When we evaluated the contacts of our patients through standard contact-tracing interviews, we found that only one patient (\#9) had a contact with a laboratory-confirmed SARS-CoV-2 subject and developed the typical COVID-19 symptomatology.

\section{Discussion}

The coronaviruses are known to be associated with the development of SAT, and an apparent increase of patients diagnosed with SAT during the COVID-19 pandemic has been reported [4]. However, to achieve the proof of a direct relationship between SARS-CoV-2 and SAT remains challenging [4]. To the best of our knowledge, here we reported the first series of SAT diagnosed during the COVID-19 pandemic in which both SARS-CoV-2-related symptoms and contact-tracing data were collected. Among our SAT patients, only one presented a typical clinical presentation of SARS-CoV-2 with persistence of fatigue over the following five months and had a close contact with a SARS-CoV-2infected individual just before she developed SAT. However, she underwent diagnostic tests for SARS-CoV-2 (rtPCR in nasopharyngeal swab and serum) with negative results. In all the other patients, the symptomatology was not typical for SARS-CoV-2 and no positive SARS-CoV-2 tests were recorded. Overall, it was difficult in our series to demonstrate a clinical relationship between SAT and SARS-CoV-2, while one case remained clinically suspicious for SARSCoV-2 infection. To date, a small number of SAT patients with proven SARS-CoV-2 have been published [8-13]. All those cases had mild SARS-CoV-2 clinical presentation except one [10] and had a typical clinical course of SAT. According to the published cases, it seems that some patients with SARS-CoV-2 can be affected by SAT and this should be taken into account also in those patients with mild SARSCoV-2 manifestation. Interestingly, no patient of our series was placed in quarantine because of the lack of proof of SARS-CoV-2 infection, but nobody of their close contacts (i.e., family members, cohabitants, and colleagues) developed SARS-CoV-2-related symptoms. Therefore, SAT could not be considered as related to SARS-CoV-2 until proven otherwise.

SAT is presumed to be associated with viral infection or postviral inflammation. Indeed, a possible epidemiological linkage between enterovirus and thyroiditis has been described [5, 14]. Moreover, herpes viruses (e.g., cytomegalovirus and Epstein-Barr virus) have been considered in the pathogenesis of SAT [15-17]. However, it is an open issue whether they are responsible for thyroid diseases or there is only an epidemiological linkage based on seasonality or coincident increase of indirect markers (e.g., antibodies). It 
TABLE 1: Clinical symptoms and contact-tracing data of the included patients.

\begin{tabular}{|c|c|c|c|c|c|c|c|c|c|c|c|c|c|}
\hline & Age & Gender & Fever & Cough & Dyspnea & Headache & Fatigue & Pneumonia & $\begin{array}{l}\text { Loss } \\
\text { of } \\
\text { smell }\end{array}$ & $\begin{array}{l}\text { Loss } \\
\text { of } \\
\text { taste }\end{array}$ & $\begin{array}{c}\text { SARS-CoV-2 } \\
\text { nasopharyngeal } \\
\text { swab }\end{array}$ & $\begin{array}{l}\text { SARS- } \\
\text { CoV-2 } \\
\text { serum } \\
\text { test }\end{array}$ & $\begin{array}{c}\text { Contact with } \\
\text { other } \\
\text { individuals } \\
\text { proved to be } \\
\text { positive for } \\
\text { SARS-CoV-2 }\end{array}$ \\
\hline 1 & 36 & $\mathrm{~F}$ & - & - & - & - & c & - & - & - & - & - & - \\
\hline 2 & 53 & $\mathrm{~F}$ & - & - & - & - & $\mathrm{a}$ & - & - & - & - & $\begin{array}{l}\text { b } 2 x \\
\text { neg }\end{array}$ & - \\
\hline 3 & 58 & M & - & - & - & - & - & - & - & - & - & - & - \\
\hline 4 & 44 & $\mathrm{~F}$ & $\begin{array}{c}c \\
(38.5)\end{array}$ & - & - & - & - & - & - & - & c $1 x$ neg & - & - \\
\hline 5 & 54 & $\mathrm{~F}$ & $\begin{array}{c}c \\
(38.5)\end{array}$ & - & $\mathrm{b}$ & c & - & - & - & - & - & - & - \\
\hline 6 & 44 & $\mathrm{~F}$ & $\begin{array}{c}c \\
(38.5)\end{array}$ & c & - & - & $\mathrm{a}$ & - & - & - & c $2 x$ neg & - & - \\
\hline 7 & 66 & $\mathrm{~F}$ & - & - & - & - & c & - & - & - & - & - & - \\
\hline 8 & 46 & $\mathrm{~F}$ & - & - & - & - & - & - & - & - & - & - & - \\
\hline 9 & 39 & $\mathrm{~F}$ & $\begin{array}{c}c \\
(38.0)\end{array}$ & c & c & & $\mathrm{a}$ & - & $\mathrm{a}$ & $\mathrm{a}$ & a $1 x$ neg & $\begin{array}{l}\text { b } 2 x \\
\text { neg }\end{array}$ & $\begin{array}{c}\text { Yes, just } \\
\text { before SAT }\end{array}$ \\
\hline 10 & 33 & $\mathrm{~F}$ & $\begin{array}{c}\mathrm{c} \\
(37.3) \\
\end{array}$ & - & - & - & - & - & - & - & b 2x neg & - & - \\
\hline
\end{tabular}

"a", "b," and "c" indicate if the symptom or feature was after, before, or concomitant to the SAT, respectively; the highest body temperature is indicated in parenthesis and is expressed in Celsius scale; "neg" means negative test; "2x" indicates repeated tests. The empty cells indicate the absence of the symptom or feature.

is reasonable that COVID-19 may influence the burden of SAT. SARS-CoV-2, probably due to its immunosuppressive effects in severe cases (i.e., lymphopenia [18]), may represent a risk factor for herpes viruses reactivation [19], thus leading to a subsequent thyroiditis. Moreover, it is possible that the inflammatory response produced by SARS-CoV-2 may influence further inflammation damages in thyroid follicles. All these pathogenic considerations, however, were speculative and our analysis appears to mitigate the probability of a clear association between SARS-CoV-2 infection and SAT. Further studies are needed to elucidate this association. For example, a casecontrol (i.e., including SAT patients and matched controls) study assessing serological response to COVID-19 may be a valuable option to unmask a possible association between SARS-CoV-2 and SAT. Such a study should be performed ideally before the broad introduction of SARS$\mathrm{CoV}-2$ vaccines. A direct cytopathic role of SARS-CoV-2, which appears to be unlikely, would necessitate the identification of viable virus in thyroid gland specimens.

Some specific considerations about our series of SAT have to be addressed. The present series was collected in a region (Canton Ticino, Southern Switzerland) which was particularly affected by COVID-19 pandemic with about 18,000 cases and 650 deaths until mid-December 2020 in a population of 350,000 inhabitants [20,21]. In this Canton, a lockdown was implemented in the spring of 2020 (with complete stop of nonurgent thyroid visits in the regional hospitals) [22]. The patients were followed up at one single clinic and it was not possible to compare the frequency of SAT recorded during the study period with that of the previous year. A half of our patients did not undergo nasopharyngeal swab or serum test (see Table 1) and we do not have a solid proof in favor or against their SARS-CoV-2 infection. Most our cases were diagnosed during spring and summer, and a seasonal effect should be considered [14]. The possibility of false negative SARS-CoV-2 diagnostic tests, especially in asymptomatic patients, should be taken into account.

In conclusion, the present study showed that most patients diagnosed with SAT during COVID-19 pandemic rarely experienced the typical symptoms and signs of SARS$\mathrm{CoV}-2$. Indeed, according to contact tracing, only one patient met individuals diagnosed with SARS-CoV-2. These data do not exclude that SAT is an underestimated manifestation of SARS-CoV-2 and further studies are needed to clarify their possible association. Considering the rapid evolution of research on COVID-19, the present findings might be revised soon.
Abbreviations
COVID-19: Coronavirus disease 2019
SARS- Severe acute respiratory syndrome
CoV-2: $\quad$ coronavirus 2
SAT: $\quad$ Subacute thyroiditis.

\section{Data Availability}

The retrospective, blinded, grouped data used to support the findings of this study are available from the corresponding author upon request. 


\section{Ethical Approval}

All procedures performed in studies involving human participants were in accordance with the institutional ethical standards and in accordance with the Declaration of Helsinki of 1964 and its later amendments or comparable ethical standards. The institutional rules should not oblige authors using retrospective data of patients in anonymous and grouped manner to specific process. Patients included in the present study gave informed consent to publish their data.

\section{Consent}

Informed consent was obtained from all individual participants included in the study.

\section{Conflicts of Interest}

The authors declare that they have no conflicts of interest.

\section{Authors' Contributions}

Pierpaolo Trimboli conceptualized the study; Pierpaolo Trimboli and Niccolò Buetti developed methodology; Pierpaolo Trimboli and Chiara Camponovo did formal analysis and investigation; Pierpaolo Trimboli and Niccolò Buetti wrote the original draft; Sebastiano Franscella and Enos Bernasconi reviewed and edited the article.

\section{References}

[1] WHO, "Director-General's opening remarks at the media briefing on COVID-19," 2020, https://www.who.int/dg/ speeches/detail/who-director-general-s-opening-remarks-atthe-media-briefing-on-covid-19---11-march-2020.

[2] 2020 https://gisanddata.maps.arcgis.com/apps/opsdashboard/ index.html\#/bda7594740fd40299423467b48e9ecf6.

[3] L. Wei, S. Sun, C.-H. Xu et al., "Pathology of the thyroid in severe acute respiratory syndrome," Human Pathology, vol. 38, no. 1, pp. 95-102, 2007.

[4] L. Scappaticcio, F. Pitoia, K. Esposito, A. Piccardo, and P. Trimboli, "Impact of COVID-19 on the thyroid gland: an update," Reviews in Endocrine and Metabolic Disorders, pp. 1-13, 2020.

[5] R. Desailloud and D. Hober, "Viruses and thyroiditis: an update," Virology Journal, vol. 6, no. 1, p. 5, 2009.

[6] E. N. Pearce, A. P. Farwell, and L. E. Braverman, "Thyroiditis," New England Journal of Medicine, vol. 348, no. 26, pp. 2646-2655, 2003.

[7] M. Rotondi, F. Coperchini, G. Ricci et al., "Detection of SARSCOV-2 receptor ACE-2 mRNA in thyroid cells: a clue for COVID-19-related subacute thyroiditis," Journal of Endocrinological Investigation, pp. 1-6, 2020.

[8] A. Brancatella, D. Ricci, N. Viola, D. Sgrò, F. Santini, and F. Latrofa, "Subacute thyroiditis after sars-CoV-2 infection," Journal of Clinical Endocrinology and Metabolism, vol. 105, no. 7, p. dgaa276, 2020.

[9] A. Brancatella, D. Ricci, D. Cappellani et al., "Is subacute thyroiditis an underestimated manifestation of SARS-CoV-2 infection? insights from a case series," Journal of Clinical Endocrinology and Metabolism, vol. 105, no. 10, p. dgaa537, 2020.
[10] S. Ippolito, F. Dentali, and M. L. Tanda, "SARS-CoV-2: a potential trigger for subacute thyroiditis? Insights from a case report," Journal of Endocrinological Investigation, vol. 43, no. 8, pp. 1171-1172, 2020.

[11] E. Asfuroglu Kalkan and I. Ates, "A case of subacute thyroiditis associated with COVID-19 infection," Journal of Endocrinological Investigation, vol. 43, no. 8, pp. 1173-1174, 2020.

[12] R. M. Ruggeri, A. Campenni, M. Siracusa, G. Frazzetto, and D. Gullo, "Subacute thyroiditis in a patient infected with SARS-COV-2: an endocrine complication linked to the COVID-19 pandemic," Hormones (Athens), vol. 20, no. 1, pp. 219-221, 2020.

[13] S. A. M. Mattar, S. J. Q. Koh, S. Rama Chandran, and B. P. Z. Cherng, "Subacute thyroiditis associated with COVID-19," BMJ Case Reports, vol. 13, no. 8, p. e237336, 2020.

[14] E. Martino, L. Buratti, L. Bartalena et al., "High prevalence of subacute thyroiditis during summer season in Italy," Journal of Endocrinological Investigation, vol. 10, no. 3, pp. 321-323, 1987.

[15] A. Espino Montoro, M. Medina Perez, M. C. Gonzalez Martin, R. Asencio Marchante, and J. Lopez Chozas, "Subacute thyroiditis associated with positive antibodies to the EpsteinBarr virus," Anales de Medicina Interna, vol. 17, no. 10, pp. $546-548,2000$.

[16] C. Volta, N. Carano, M. E. Street, and S. Bernasconi, "Atypical subacute thyroiditis caused by epstein-barr virus infection in a three-year-old girl," Thyroid, vol. 15, no. 10, pp. 1189-1191, 2005.

[17] A. Al Maawali, S. Al Yaarubi, and A. Al Futaisi, "An infant with cytomegalovirus-induced subacute thyroiditis," Journal of Pediatric Endocrinology and Metabolism, vol. 21, no. 2, pp. 191-193, 2008.

[18] L. M. Khosroshahi and N. Rezaei, "Dysregulation of the immune response in COVID-19," Cell Biology International, 2020.

[19] P. Le Balc'h, K. Pinceaux, C. Pronier, P. Seguin, J. M. Tadié, and F. Reizine, "Herpes simplex virus and cytomegalovirus reactivations among severe COVID-19 patients," Critical Care, vol. 24, no. 1, p. 530, 2020.

[20] 2020 https://www4.ti.ch/dss/dsp/covid19/home.

[21] N. Buetti, P. Trimboli, T. Mazzuchelli et al., "Diabetes mellitus is a risk factor for prolonged SARS-COV-2 viral shedding in lower respiratory tract samples of critically ill patients," Endocrine, vol. 70, no. 3, pp. 454-460, 2020.

[22] A. Smulever, E. Abelleira, F. Bueno, and F. Pitoia, "Thyroid cancer in the era of COVID-19," Endocrine, vol. 70, no. 1, pp. 1-5, 2020. 